\title{
Acute Tropical Pulmonary Eosinophilia
}

\author{
Characterization of the Lower Respiratory Tract Inflammation and Its Response to Therapy
}

Paula Pinkston, V. K. Vijayan, Thomas B. Nutman, William N. Rom, Kathleen M. O'Donnell, Mary J. Comelius, Y. Kumaraswami, V. J. Ferrans, T. Takemura, Gordon Yenokida, K. V. Thiruvengadam, S. P. Tripathy,

Éric A. Ottesen, and Ronald G. Crystal

Pulmonary Branch and Pathology Branch, National Heart, Lung, and Blood Institute and Laboratory of Parasitic Diseases, National Institute of Allergy and Infectious Diseases, National Institutes of Health, Bethesda, Maryland 20892;

Tuberculosis Research Center, Indian Council of Medical Research, Madras, India

\begin{abstract}
Although acute tropical pulmonary eosinophilia (TPE) is well recognized as a manifestation of filarial infection, the processes that mediate the abnormalities of the lung in TPE are unknown. To evaluate the hypothesis that the derangements of the lower respiratory tract in this disorder are mediated by inflammatory cells in the local milieu, we utilized bronchoalveolar lavage to evaluate affected individuals before and after therapy. Inflammatory cells recovered from the lower respiratory tract of individuals with acute, untreated TPE $(n=8)$ revealed a striking eosinophilic alveolitis, with marked elevations in both the proportion of eosinophils (TPE 54 $\pm 5 \%$; normal $2 \pm 5 \%$; $P<0.001$ ) and the concentration of eosinophils in the recovered epithelial lining fluid (ELF) (TPE $63 \pm 20 \times 10^{3} / \mu \mathrm{l}$; normal $0.3 \pm 0.1$ $\left.\times 10^{3} / \mu \mathrm{l} ; P<0.01\right)$. Importantly, when individuals $(n=5)$ with acute TPE were treated with diethylcarbamazine (DEC), there was a marked decrease of the lung eosinophils and concomitant increase in lung function. These observations are consistent with the concept that at least some of the abnormalities found in the lung in acute TPE are mediated by an eosinophil-dominated inflammatory process in the lower respiratory tract.
\end{abstract}

\section{Introduction}

Tropical pulmonary eosinophilia (TPE) ${ }^{1}$ is an interstitial lung disease that results from a heightened immunologic response to the human filarial parasites, Wuchereria bancrofti and Brugia malayi (1). TPE has been reported from filarial endemic regions all over the world, but it is especially prevalent in India and Southeast Asia $(1,2)$. For reasons that are not yet clear, individuals with TPE respond to filarial infections with vigorous immune responses and severe clinical manifestations that contrast markedly with those of most individuals living in the filarial endemic region, who often have filarial infections but commonly show highly suppressed immunologic responses and minimal clinical reactions (2-4).

Individuals with acute TPE characteristically present with

Address reprint requests to Dr. Crystal, Building 10, Room 6D03, NIH, Bethesda, MD 20892. 1986

Received for publication 2 June 1986 and in revised form 17 December

1. Abbreviations used in this paper: DEC, diethylcarbamazine; ELF, epithelial lining fluid; $\mathrm{FEV}_{1}$, forced expiratory volume in $1 \mathrm{~s}$; TPE, tropical pulmonary eosinophilia.

The Journal of Clinical Investigation, Inc.

Volume 80, July 1987, 216-225 cough, dyspnea, nocturnal wheezing, and occasionally fever, anorexia, and weight loss. Their chest $\mathrm{x}$-rays have diffuse reticulonodular infiltrates, and pulmonary function tests show primarily restrictive defects with mild obstruction $(1,5,6)$. Unlike results obtained from normals and individuals with other manifestations of filarial infection, laboratory studies from individuals with acute TPE demonstrate marked peripheral blood eosinophilia and high serum concentrations of IgE and filarial-specific IgG and IgE antibodies (1-5). Although most individuals with acute TPE have a rapid clinical response to a standard course of diethylcarbamazine (DEC), with reduced cough and dyspnea, in some individuals the pulmonary disease progresses to a chronic form that results in interstitial fibrosis and permanent loss of lung function (6-9).

The current hypothesis concerning the pathogenesis of TPE suggests that it begins with a lung parenchymal inflammation in persons highly sensitized immunologically to filarial parasites. This inflammation is initiated by microfilariae that have been released from lymphatic dwelling adult worms into the circulation and cleared in the pulmonary vasculature. According to this concept, these trapped microfilariae degenerate and release their antigenic constituents that trigger local inflammatory and immune processes $(9-11)$. Consistent with this concept, very limited morphologic data has revealed evidence of degenerating microfilariae and diffuse inflammation in the lung parenchyma $(6,9,10,12,13)$.

In this context, it is reasonable to hypothesize that the clinical and physiologic pulmonary abnormalities characteristic of TPE are mediated, at least in part, by the accumulation of inflammatory cells in the lung parenchyma. As a first step in defining the inflammatory processes, we have utilized the technique of bronchoalveolar lavage to sample and analyze the inflammatory cells from the lower respiratory tract of individuals with acute TPE and to correlate the status of this inflammation with the changes in lung function observed following therapy with DEC.

\section{Methods}

\section{Study population}

All individuals in the study population were residents of the area surrounding Madras, India, a region endemic for bancroftian filariasis. Clinical evaluation of each individual included detailed history, physical examination, chest $\mathrm{x}$-rays, pulmonary function tests (using P. K. Morgan pulmonary function test apparatus), and blood and stool examinations for the presence of other parasites. To determine the immunologic status of the study population to filaria, each individual had total serum IgE measured by a standard radioimmunosorbant technique (14) (Pharmacia Fine Chemicals, Piscataway, NJ) and specific IgG filarial antibody determined by an enzyme immunoassay (15) using antigen prepared from Brugia malayi adult worms (14). 
Individuals with acute TPE. The diagnosis of acute TPE was established in eight individuals based on the accepted criteria (1) of residence in the endemic area of southern India, recent onset of symptoms, chest $x$-ray infiltrates, lung function abnormalities, peripheral eosinophilia, high total serum IgE, and high serum titers of antifilarial IgG (Table I). In addition, two individuals complained of fever and weight loss.

Normals. Seven normal nonsmokers from the Madras area were evaluated as controls (Table I). None had respiratory symptoms or abnormal physical findings, and all had normal chest $\mathrm{x}$-rays and normal pulmonary function tests. None had elevated peripheral blood eosinophil counts, high serum concentrations of total IgE, or high concentrations of filarial-specific IgG.

Individuals with elephantiasis. Four individuals who had elephantiasis, a manifestation of filarial infection with no pulmonary involvement, were also evaluated (Table I). Except for the chronic problem of elephantiasis, all individuals were asymptomatic at the time of evaluation, with normal chest examinations, chest $\mathrm{x}$-rays and pulmonary function tests. Mean blood eosinophil counts were normal, but there were mildly elevated serum IgE levels. Antifilarial IgG antibody titers were distinctly lower than those of the TPE patients.

Individuals with asthma. Since acute TPE and asthma are both characterized by cough, dyspnea, wheezing, and blood eosinophilia, four individuals with asthma but no evidence of filarial infection were also evaluated (Table I). The diagnosis of asthma was based on a history of wheezing, exertional dyspnea, and clinical response to therapy with oral bronchodilators. All had mild blood eosinophilia, but total IgE and filariaspecific IgG levels were markedly lower than those associated with TPE. Three had cough, dyspnea, and wheezing, and two had wheezing on physical examination. When evaluated, all had normal pulmonary function tests. One individual had a chest $\mathrm{x}$-ray showing a scattered mild nodular pattern; however, this individual had normal pulmonary function tests and normal bronchoalveolar lavage findings.

\section{Collection of lung effector cells from the lower respiratory tract}

All studies were carried out under protocols approved by the National Institutes of Health, USA, and the Indian Council of Medical Research, India. In all cases, the procedures to be performed were explained in English or the local language by a member of the staff and consent was obtained.

Bronchoscopy and bronchoalveolar lavage were performed under local anesthesia without complications in the outpatient facility of the Tuberculosis Research Center, Madras, India. Since individuals in two of the study groups, acute TPE and asthma, were predisposed to bronchospasm, all individuals, except for the normal subjects, received intravenous aminophylline $(0.5 \mathrm{mg} / \mathrm{ml}$ at $100 \mathrm{ml} / \mathrm{h})$ before, during, and after the procedure. One individual in the acute TPE group and one individual in the asthma group developed moderate bronchospasm following the procedure; in both cases, this was easily treated with additional intravenous aminophylline, inhaled isoproterenol, and nasal oxygen. All individuals were observed for $3 \mathrm{~h}$ after bronchoscopy and then discharged from the clinic. When reevaluated $24 \mathrm{~h}$ later, one individual complained of a transient fever the evening following bronchoscopy, but, otherwise, there were no significant complications from the procedure.

Lung effector cells from the lower respiratory tract were obtained by bronchoalveolar lavage with $300 \mathrm{ml}$ of saline, as previously described (19). Briefly, $100 \mathrm{ml}$ of sterile saline in five $20-\mathrm{ml}$ aliquots was infused through a fiberoptic bronchoscope into each of three lobes in the lower respiratory tract. The cells and lavage fluid were recovered by gentle suction after each aliquot was infused. The cells recovered by lavage were counted on a hemocytometer, and lavage cell differentials were determined using filter preparations stained with hematoxylin and eosin (20).

\section{Evaluation of the concentration of eosinophils in lung and blood}

To estimate the relative concentration between eosinophils in the lower respiratory tract and in the blood, the total numbers of eosinophils re-

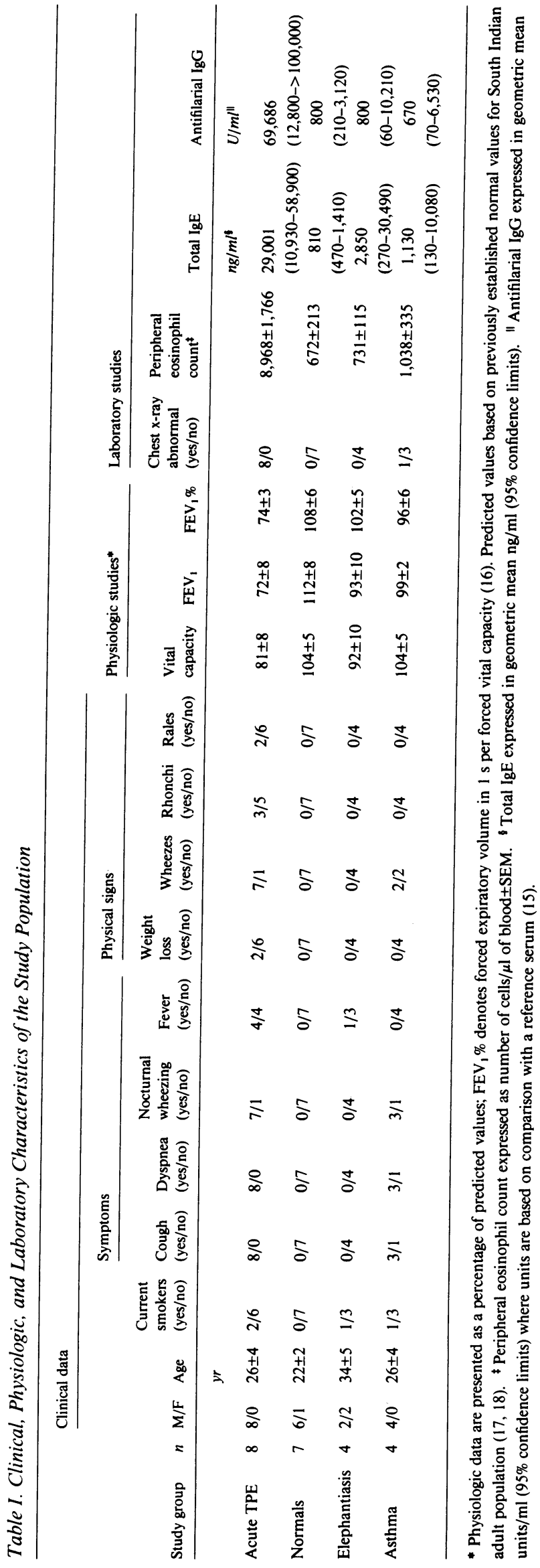


moved by bronchoalveolar lavage were referenced to the amount of the total lower respiratory tract epithelial lining fluid (ELF) recovered in the same procedure. The amount of ELF recovered was estimated using urea as an internal standard, as previously described (21). The percent recovery of the lavage fluid from the various groups were: normals $60 \pm 4 \%$, acute TPE $49 \pm 1 \%$, asthma $40 \pm 7 \%$, and elephantiasis $60 \pm 4 \%$.

\section{Evaluation of the morphology of eosinophils}

To evaluate the morphology of the eosinophils, blood and lung cells were pelleted by centrifugation, fixed with $3 \%$ glutaraldehyde in $0.1 \mathrm{M}$ phosphate buffer, $\mathrm{pH} 7.2$, washed with several changes of buffer, postixed with $1 \%$ osmium tetroxide in phosphate buffer, dehydrated with ethanol and propylene oxide and embedded in Polybed 812. Ultrathin sections were stained with uranyl acetate and lead citrate and examined with a transmission electron microscope at an accelerating voltage of $80 \mathrm{kV}$. Evaluation of the eosinophil granule crystallized core and matrix was performed on 100 randomly chosen eosinophils per study subject.

\section{Short-term effects of treatment on acute TPE}

To determine the short-term effects of therapy on the alveolitis of acute TPE, five of the individuals with acute TPE were reevaluated by bronchoalveolar lavage 6-12 d after starting therapy with DEC $(6 \mathrm{mg} / \mathrm{kg}$ per d). To determine if changes in lung function accompanied changes in the alveolitis associated with therapy, pulmonary function tests were carried out before the repeat lavage procedures. To evaluate the longterm results of a standard 3-wk course of therapy, three of these individuals were reevaluated one year later by bronchoalveolar lavage and lung function tests.

\section{Data analysis}

All data except quantitation of immunoglobulins (Table I) are presented as mean \pm SEM. Since $\log$-transformed serum IgE values are normally distributed, the results for total serum IgE (Table I) are presented as the geometric mean with $95 \%$ confidence limits. Likewise, the antifilarial IgG values (Table I) are presented as the geometric mean with $95 \%$ confidence limits, as previously reported (11).

All results between groups were compared using the two-tailed Student's $t$ test; paired results from individuals were compared using the paired $t$ test. Paired results before and after therapy were compared in terms of percent change using the one sample $t$ test.

\section{Resuilts}

\section{Characterization of lung inflammatory cell population in acute TPE}

Acute TPE was associated with an intense alveolitis (Table II). The total number of inflammatory cells recovered from the lavage fluid of individuals with acute TPE $\left(241 \pm 38 \times 10^{6}\right)$ was approximately fourfold greater than that of any of the three control populations (total number of cells recovered from normals,
$34 \pm 7 \times 10^{6}$; patients with elephantiasis, $66 \pm 15 \times 10^{6}$; and asthmatics, $\left.30 \pm 18 \times 10^{6}\right)$. This was true whether all individuals or only nonsmokers were considered $(P<0.05$, TPE compared to all other groups).

When referenced in terms of the total volume of epithelial lining fluid recovered by the lavage procedure, it was apparent that the increase in number of recovered inflammatory cells in acute TPE was a reflection of the markedly increased concentration of inflammatory cells in fluid lining the epithelial surface of the lower respiratory tract. In this regard, acute TPE patients had a lavage cell concentration at least four times greater than any of the control populations $(P<0.025$; Table II).

Bronchoalveolar lavage cell differentials revealed that the eosinophil was the predominant type of inflammatory cell recovered from the lavage fluid of patients with acute TPE (Table II, Fig. 1). There were no significant differences in the percentages of recovered lymphocytes or neutrophils among normal individuals or those with acute TPE, elephantiasis, or asthma. In marked contrast, the percentage of eosinophils recovered from individuals with acute TPE was dramatically increased compared to that of all other groups $(P<0.001$, TPE compared to all others). As a consequence, the percentage of macrophages recovered in the acute TPE group was proportionately reduced compared to that of the other clinical groups $(P<0.005)$. There were no unusual cell types such as basophils, mast cells, or plasma cells recovered from the lower respiratory tract of any individual studied, and epithelial cells always represented $<5 \%$ of the cells recovered. Furthermore, there were no microfilariae identified in the lavage fluid or on the filter preparations.

Evaluation of the concentration of each cell type in the recovered ELF demonstrated that the concentration of eosinophils in individuals with acute TPE $\left(63 \pm 20 \times 10^{3}\right.$ eosinophils/ $/ \mu$ ELF $)$ was at least 21-fold greater than that of any other group. In contrast, the concentration of lymphocytes, neutrophils, and macrophages was similar, although the TPE patients had a mild increase in neutrophils, among all study groups $(P>0.2)$. Thus, the pulmonary inflammation of acute TPE was characterized by an intense eosinophilic alveolitis demoristrated by marked increases in both the percentage of eosinophils recovered by lavage and in the concentration of eosinophils in the epithelial lining fluid of the lower respiratory tract.

\section{Relative concentration of eosinophils in peripheral blood and lung $E L F$}

When direct comparisons of lung and blood eosinophil concentrations were made, it was apparent that the individuals with acute TPE had a 3- to 20-fold greater concentration of eosinophils

Table II. Analysis of Cells and Fluid Recovered by Bronchoalveolar Lavage

\begin{tabular}{|c|c|c|c|c|c|c|c|c|c|c|}
\hline \multirow[b]{2}{*}{ Study group } & \multirow[b]{2}{*}{$\mathbf{n}$} & \multicolumn{4}{|c|}{ Cell differential* } & \multicolumn{5}{|l|}{ Cell density* } \\
\hline & & $\mathbf{M}$ & $\mathbf{L}$ & $\mathbf{N}$ & $\mathbf{E}$ & $\begin{array}{l}\left.\text { [Total cells } \times 10^{3}\right] / \\
{[\mu 1 \text { ELF] }}\end{array}$ & $\begin{array}{l}{\left[\mathrm{M} \times 10^{3}\right] /} \\
{[\mu \mathrm{l} E L F]}\end{array}$ & $\begin{array}{l}{\left[\mathrm{L} \times 10^{3}\right] /} \\
{[\mu \mathrm{L} \text { ELF }]}\end{array}$ & $\begin{array}{l}{\left[N \times 10^{3}\right] /} \\
{[\mu \text { ELF }]}\end{array}$ & $\begin{array}{l}{\left[\begin{array}{lll}E & \left.\times 10^{3}\right] / \\
{[\mu l} & E L F\end{array}\right]}\end{array}$ \\
\hline Acute TPE & 8 & $32 \pm 5$ & $10 \pm 2$ & $4 \pm 3$ & $54 \pm 5$ & $88 \pm 17$ & $27 \pm 6$ & $8 \pm 2$ & $5 \pm 3$ & $47 \pm 11$ \\
\hline Normals & 7 & $76 \pm 4$ & $21 \pm 4$ & $2 \pm 0$ & $2 \pm 0$ & $20 \pm 6$ & $22 \pm 6$ & $4 \pm 1$ & $<1$ & $<1$ \\
\hline Elephantiasis & 4 & $72 \pm 6$ & $25 \pm 5$ & $1 \pm 0$ & $3 \pm 1$ & $25 \pm 6$ & $16 \pm 7$ & $5 \pm 0$ & $<1$ & $<1$ \\
\hline Asthma & 4 & $66 \pm 4$ & $22 \pm 6$ & $2 \pm 1$ & $10 \pm 4$ & $19 \pm 9$ & $13 \pm 6$ & $4 \pm 1$ & $<1$ & $3 \pm 2$ \\
\hline
\end{tabular}

* Cell differentials on filter preparations; macrophages $(M)$, lymphocytes $(L)$, neutrophils $(N)$ and eosinophils $(E)$. ${ }^{\ddagger}$ Cell density determined as [number of cells of each type recovered]/[amount of ELF recovered]; ELF is epithelial lining fluid (21). 


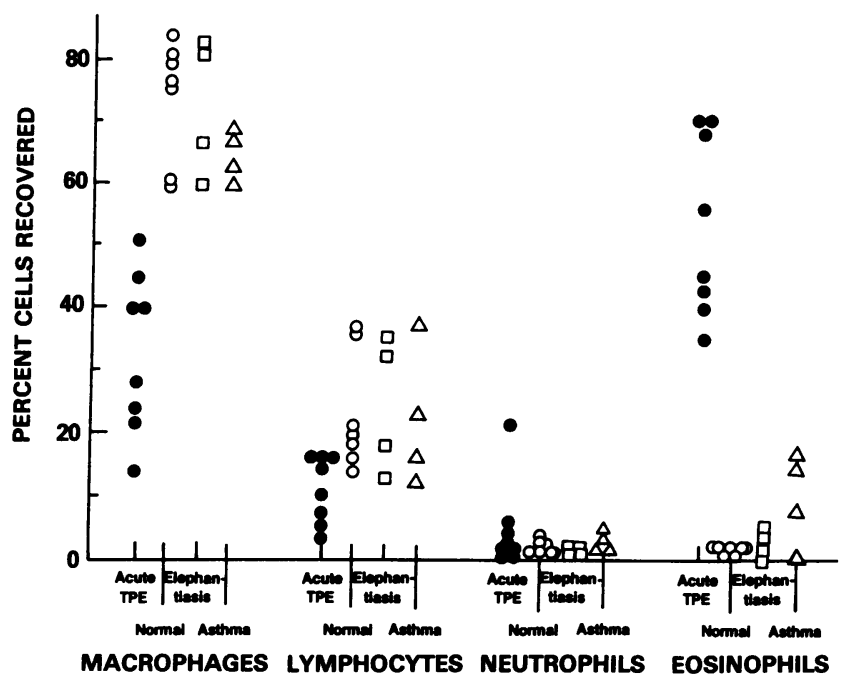

Figure 1. Bronchoalveolar lavage cell differentials from individuals with acute TPE (๑), normals (0), individuals with elephantiasis ( $\square$ ), and individuals with asthma $(\Delta)$. The percentage of each cell type recovered is represented for each individual in the various groups.

in their lung ELF than in their blood $(P<0.01)$ (Fig. 2). For other clinical groups, by contrast, there was no significant difference between the concentration of eosinophils in lung and blood ( $P>0.2$, all comparisons). Since the concentration of eosinophils in ELF was much greater than would be expected from passive infiltration of peripheral blood eosinophils into the lungs, these observations suggest that the lung eosinophilia of individuals with acute TPE represents a selective process of eosinophil accumulation in the lower respiratory tract.

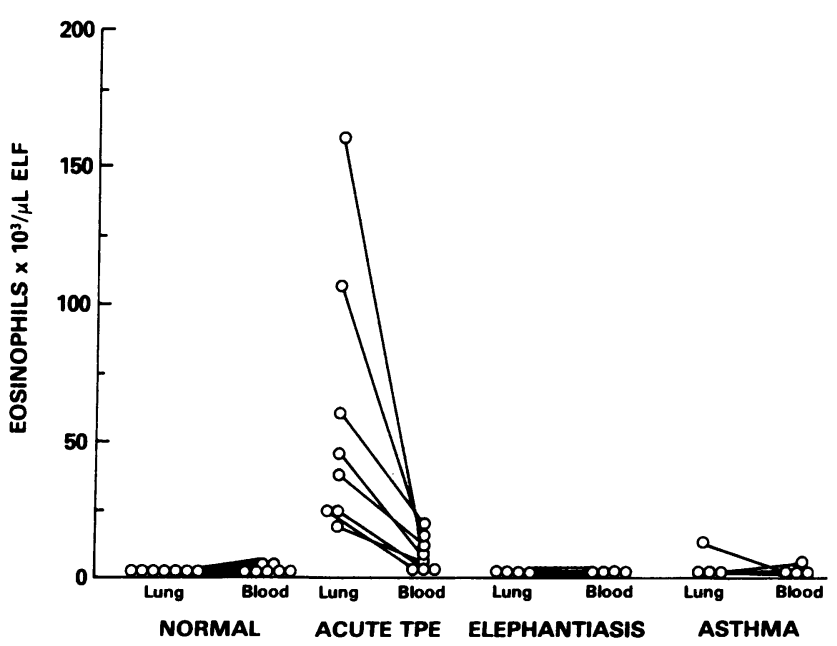

Figure 2. Comparison of the concentration of eosinophils in ELF of the lower respiratory tract to that of blood of individuals in the study groups. Shown are data from normals, individuals with acute TPE, individuals with elephantiasis, and individuals with asthma. The concentration of lung eosinophils in ELF is expressed as the number of eosinophils per microliter of epithelial lining fluid recovered by bronchoalveolar lavage. The concentration of blood eosinophils was determined by quantitation of the number of eosinophils contained per microliter of blood for the same individuals. The data for lung and blood of each individual are indicated as -O-

\section{Morphological changes of eosinophils}

Electron microscopic examination disclosed marked alterations in the morphology of eosinophils in the lavage fluid recovered from individuals with acute tropical eosinophilia. In comparison with eosinophils from simultaneously processed blood and lavage fluid from normal individuals (Fig. $3 A, B$ ), the lung eosinophils from individuals with acute tropical eosinophilia (Fig. $3 C-G$ ) showed marked alterations which consisted of severe degranulation with loss of both the cores and the peripheral portions of the granules. In many granules, only part of the crystalline core remained, surrounded by an electron-lucent peripheral zone (Fig. $3 \mathrm{D}$ ); the cores often also had electron-lucent central portions. Granule fusion was also present (Fig. $3 E$ ), and the granules exhibited a considerable heterogeneity of morphological changes (Fig. $3 F$ ) from empty granules to various shaped electron hypodense cores to empty or an electron hypodense periphery surrounding the cores (Fig. $3 \mathrm{G}$ ). The nuclei of these cells retained their usual morphology. The finding of marked loss of granule material correlates with the vacuolated appearance exhibited by such eosinophils in light microscopic preparations stained by the Giemsa method. The lung eosinophil granules of individuals with acute TPE had a greater loss of the crystallized core and matrix than the lung eosinophils of normals (percent lung eosinophils showing total loss of granule matrix in acute TPE $88 \pm 3 \%$, normals $48 \pm 13 \%, P<0.01$ ).

\section{Effects of treatment on the alveolitis of acute TPE}

Since acute TPE responds clinically to DEC, one would anticipate that the intense eosinophilic alveolitis would resolve following treatment. To examine this question directly, five individuals from the acute TPE group were treated with DEC and reevaluated 6-12 d later. Interestingly, even in this short period of therapy there was a profound fall in the percentage of lung eosinophils (mean decrease of $26 \pm 5 \%$; $P<0.01$; Fig. $4 \mathrm{~A}$ ). Consistent with this observation, the concentration of eosinophils in the recovered ELF also decreased in all five patients after DEC therapy (Fig. $4 \mathrm{~B}$ ). Thus, the eosinophilic alveolitis of acute TPE responded rapidly to therapy with DEC. Peripheral blood eosinophilia also fell significantly after therapy in these same five individuals $\left(8.7 \pm 1.2 \times 10^{3}\right.$ eosinophils/ $\mu$ l before to $2.2 \pm 0.5$ eosinophils $/ \mu$ l after treatment; $P<0.05$ ), but there were no immediate changes in the total serum IgE (before $30,683 \mathrm{ng} / \mathrm{ml}$, after $36,733 \mathrm{ng} / \mathrm{ml}$ ) and serum antifilarial IgG titers (before $59,200 \mathrm{U} / \mathrm{ml}$, after $70,933 \mathrm{U} / \mathrm{ml}$ ) after the first $1-2$ wk of therapy with DEC (geometric means, $P>0.2$, both comparisons).

Clinical evaluation of these same five individuals with acute TPE 6-12 d after starting DEC revealed improvement in their lung abnormalities that paralleled the resolution of the eosinophilic alveolitis. All individuals had less cough, dyspnea, nocturnal wheezing, and fever, four of the five showed improvement in the chest $\mathrm{x}$-ray abnormalities; and pulmonary function tests demonstrated increased vital capacity and $\mathrm{FEV}_{1}$ (Fig. $4 \mathrm{C}$ ). Three of the five individuals treated with DEC were available for reevaluation $1 \mathrm{yr}$ later. The proportion and concentration of eosinophils in the lung continued to decline and the lung function tests markedly improved in each individual (Fig. 4). In addition, there was a marked decline in serum titers of IgG antifilarial antibodies.

\section{Discussion}

Evaluation of individuals with acute tropical pulmonary eosinophilia demonstrated that this disease is characterized by an 

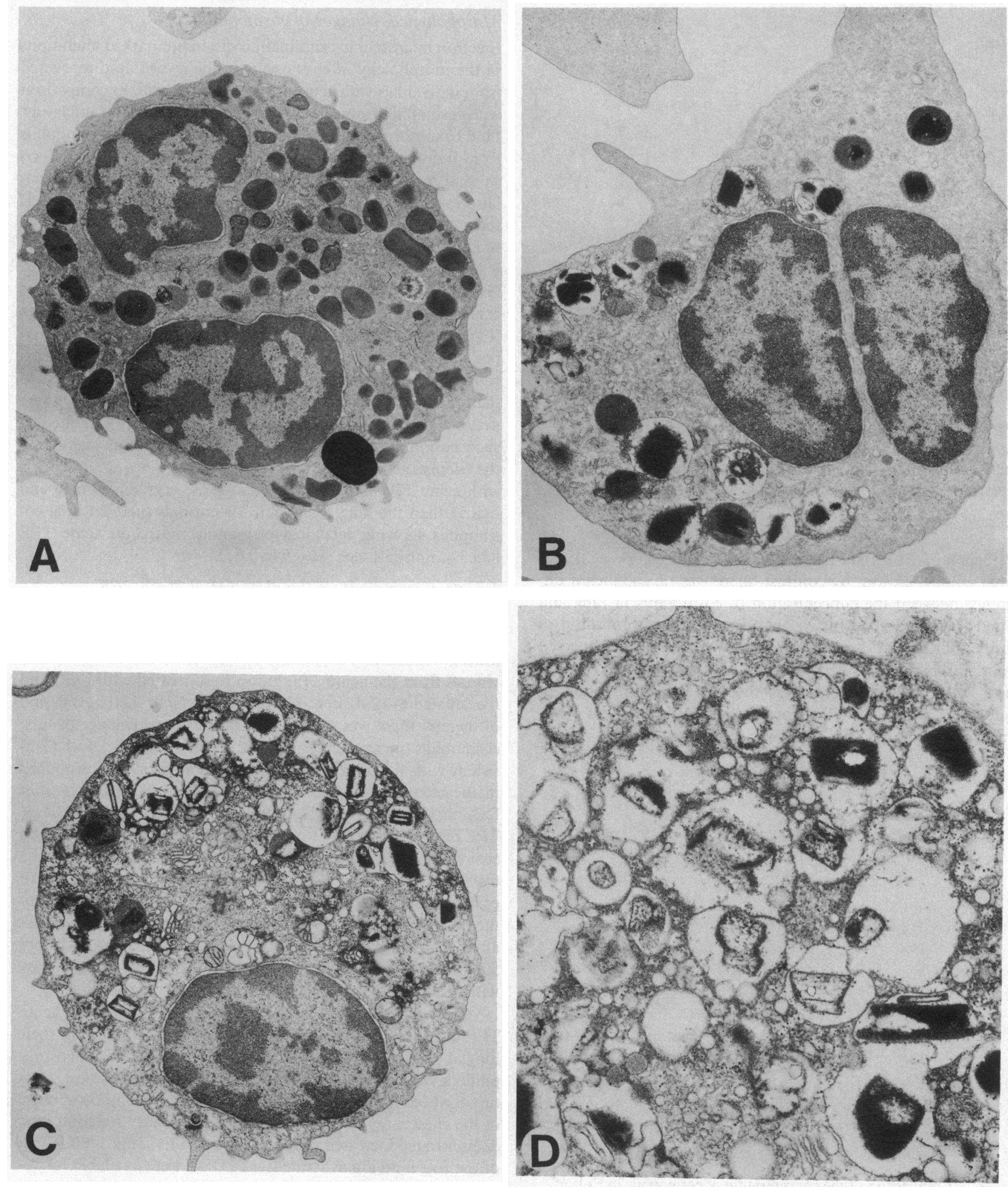

Figure 3. Electron micrographs of eosinophils from normal individuals and patients with acute tropical eosinophilia. $(A)$ Typical appearance of eosinophil in blood of a normal individual, with cytoplasmic granules that contain dense cores $(\times 11,500)$. $(B)$ Eosinophil in bronchoalveolar lavage fluid of a normal individual. The peripheral portions of some of the granules appear lucent in comparison with those of normal blood eosinophils, but the dense central cores are still evident $(\times 13,500)$. (C) Eosinophil from bronchoalveolar lavage fluid of a patient with acute tropical eosinophilia. There is marked loss of

granule content and disappearance of the dense central cores $(X 11,500)$. $(D)$ and $(E)$ Cytoplasm of eosinophils in lavage fluid of individuals with acute tropical pulmonary eosinophila. The granules show loss of central dense cores, loss of peripheral matrix, cytoplasmic vacuoles, and granule fusion $(\times 22,000)$. $(F)$ and $(G)$ High power view of cytoplasm of eosinophils in lavage fluid of individuals with acute tropical pulmonary eosinophilia. There are contrasting hypodense and dense central cores $(\times 35,000)$. 

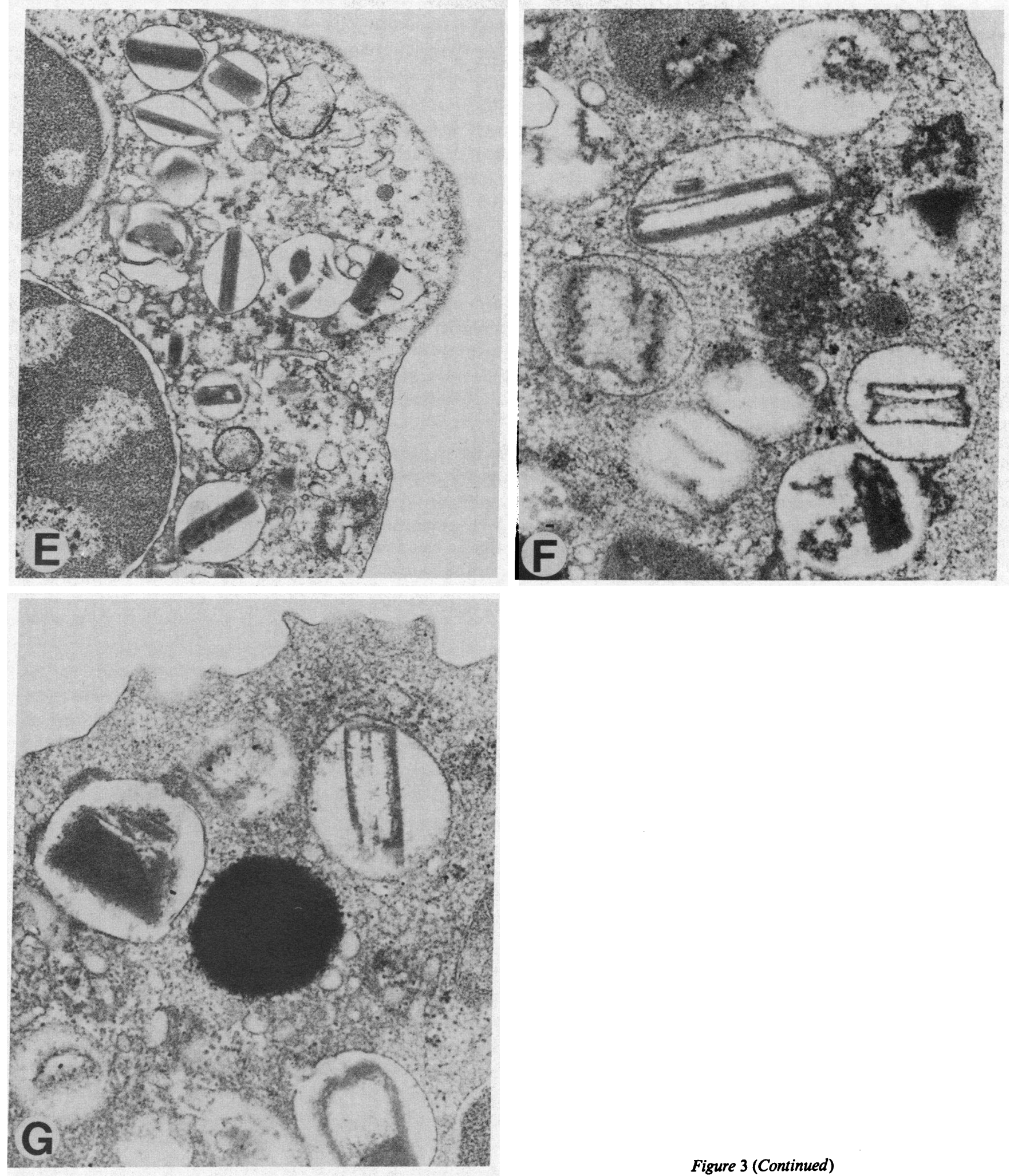

Figure 3 (Continued)

intense eosinophilic inflammatory process in the lower respiratory tract. Interestingly, while TPE is typically associated with some of the highest levels of blood eosinophilia of any human disorder (1), the concentration of eosinophils in the epithelial lining fluid of the lower respiratory tract was many fold greater than that in blood, an observation suggesting that eosinophils

accumulate selectively in the lung parenchyma. Furthermore, when these individuals were treated with DEC, the lung eosinophilia rapidly began to resolve within the first 2 wk of therapy, and this resolution was paralleled by an improvement in lung function. Importantly, reevaluation 1 yr later demonstrated that the proportion and concentration of eosinophils in both lung 

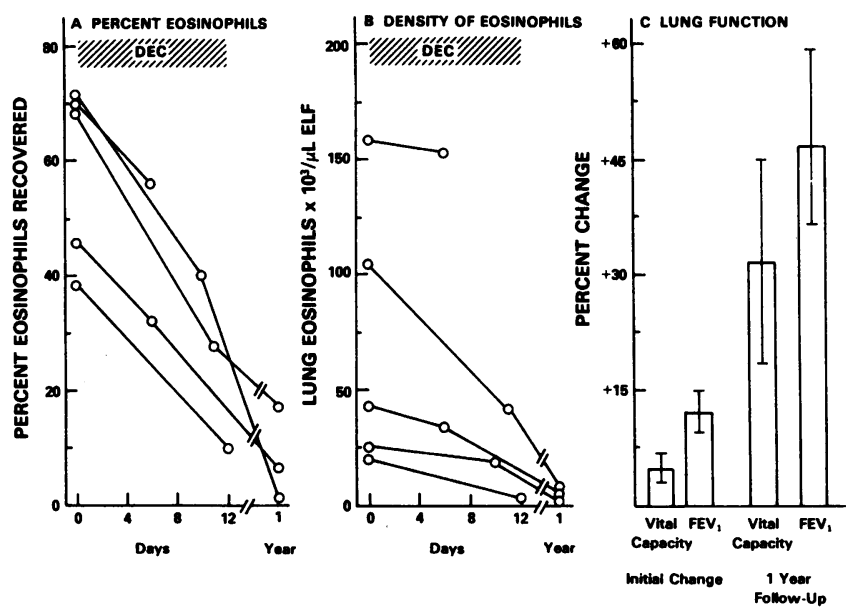

Figure 4. Effects of diethylcarbamazine on the individuals with acute TPE. Five individuals with acute TPE were evaluated by bronchoalveolar lavage and pulmonary function tests before and after therapy. Each individual was treated with DEC $(6 \mathrm{mg} / \mathrm{kg} \cdot \mathrm{d})$ and reevaluated 6 to $12 \mathrm{~d}$ after starting therapy for: $(A)$ the percentage of cells recovered by bronchoalveolar lavage that were eosinophils; $(B)$ the concentration of lung eosinophils in the epithelial lining fluid of the lower respiratory tract; and $(C)$ the change in pulmonary function tests, vital capacity and $\mathrm{FEV}_{1}$, over the same time period. Three individuals were reevaluated $1 \mathrm{yr}$ later and the same parameters were evaluated.

and blood continued to fall and the lung function continued to improve.

\section{Lower respiratory tract inflammation in acute, untreated TPE}

The observation that acute, untreated TPE is characterized by an intense eosinophilic inflammatory process in the lower respiratory tract is compatible with other clinical and morphological observations in this disease. First, although the sputum is scanty in acute TPE, the classic studies of the disease by Weingarten (22) noted that some individuals produce a sputum with clumps of eosinophils and Charcot-Leyden crystals. Second, although little data is available, the morphologic description of the lung parenchyma in acute TPE suggests that the inflammation consists mainly of eosinophils with a variable proportion of mononuclear phagocytes and lymphocytes $(6,9,10,12,13)$. Thus, it appears that the inflammatory cells recovered by lavage in acute TPE are likely representative of the overall lower respiratory tract inflammation taking place in these individuals.

In contrast to the findings in acute TPE, pulmonary eosinophils are rarely present in the normal lung, whether evaluated by bronchoalveolar lavage (19) or biopsy (23). Both light and electron microscopic studies of normal lung parenchyma (23) and studies in which normal lung parenchyma has been teased apart to sample both the interstitial and alveolar epithelial cell populations $(24,25)$ have shown that eosinophils are rarely found on the normal epithelial surface. At least two possibilities, not necessarily mutually exclusive, could explain the accumulation of eosinophils in the lung in acute TPE.

First, since these individuals have an intense blood eosinophilia, it is possible that eosinophils distribute to the lung parenchyma passively through "leaky" endothelial and epithelial cell layers. While such processes may be involved, there are many other disorders associated with blood eosinophilia in which eosinophils do not accumulate in the lung parenchyma (26). Fur- thermore, direct quantitation of the eosinophil concentration in the epithelial lining fluid recovered from the lower respiratory tract of individuals with acute TPE shows it to be at least severalfold greater than that of the eosinophil concentration in blood, i.e., there is a gradient of eosinophils from blood to lung that could not be explained by passive mechanisms and spillover from the blood.

Second, the accumulation of eosinophils in the lower respiratory tract of these individuals could potentially be modulated by eosinophil-specific chemotactic signals originating within the alveolar structures. While very little is known about such chemotactic signals specifically in the human lower respiratory tract, eosinophil-specific chemotactic factors have been described as the products of mast cells $(27,28)$, lymphocytes (29-31), and mononuclear phagocytes (32-34). Furthermore, complement components, too, can generate eosinophil chemotactic factors (35).

Regardless of the mechanism responsible for eosinophil accumulation in the acute TPE lung, it is reasonable to hypothesize that microfiliariae within the lung could be the initiating signal. In this context, serial sectioning of the few available lung biopsies from individuals with acute TPE has demonstrated degenerating microfiliariae within the lung parenchyma, often in association with eosinophils, alveolar macrophages, epithelioid cells, and foreign body giant cells $(9,10,12,13)$. These microfiliariae or their products could initiate the release of chemotactic signals secondarily from inflammatory cells or inflammatory reactants or they themselves may be chemotactic for eosinophils, as has been described for some other nematode parasites (36).

While inflammatory processes in which eosinophils dominate are very unusual in the lower respiratory tract, eosinophils have been described as contributing to the alveolitis of chronic eosinophilic pneumonia, idiopathic pulmonary fibrosis, histiocytosis $X$, hypersensitivity pneumonitis and sarcoidosis (37). In most of these cases, however, the eosinophil inflammation is mild, usually representing $<5 \%$ of the total inflammatory cell populations recovered from the lower respiratory tract (38). In addition to these interstitial lung diseases, the individuals with asthma evaluated in the present study had a small percentage of eosinophils recovered by lavage, consistent with that observed in other studies (39).

\section{Potential role of lung eosinophils in the derangement of the lower respiratory tract in TPE}

Although the mechanism by which eosinophils may be attracted to the lung in acute TPE is unknown, it is logical to consider that these cells play an important role in the pathogenesis of disease, since eosinophils have already been shown to be capable of injuring normal lung parenchymal cells, alveolar epithelial cells, fibroblasts, pleural mesothelial cells, and bronchial epithelial cells $(38,40-42)$. The mechanisms by which eosinophils do this are unclear, although oxidant radicals have been implicated (40) as has major basic protein, the primary component of the eosinophil granule core $(41,42)$. Eosinophils also carry a peroxidase capable of converting $\mathrm{H}_{2} \mathrm{O}_{2}$ to the hypohalide anion (42), which is toxic to normal cells. Furthermore, human eosinophils contain a collagenase capable of cleaving collagen types I and III, the two major collagens of the alveolar interstitium (38). In the context of TPE, it is also conceivable that the eosinophils may play a role in the bronchospasm observed in acute TPE, perhaps by virtue of the ability of these cells to release leukotrienes (41). 
Evaluation of the morphology of the eosinophils recovered from the lungs of individuals with acute TPE suggested these eosinophils were in an activated state. In this regard, the lung eosinophils showed marked degranulation, a process that follows eosinophil activation (44-57). This observation is consistent with the correlation of eosinophil activation with eosinophil degranulation observed in a variety of circumstances. For example, eosinophils lavaged from asthmatic individuals show loss of central cores and release of granules into the cytoplasm both after allergen challenges and during environmental exposure (51). In chronic eosinophilic pneumonia, eosinophils in the alveolar spaces and the interstitium have loss of granules and granule components (52-54) and macrophages from these individuals appear to have ingested alveolar eosinophil granules and CharcotLeyden crystals (55). Furthermore, major basic protein, an important component of eosinophil granules, is detected in high quantities in pleural fluid and in biopsy material in the lower respiratory tract in chronic eosinophilic pneumonia (54). In hypereosinophilic syndrome, degranulated and vacuolized blood eosinophils correlate with tissue damage including endomyocardial injury (56). In facial edema with hypereosinophilia (57) degranulated eosinophils have been found in the dermis with extracellular major basic protein localized by immunofluorescent staining. These observations, together with the knowledge that eosinophil granule components can injure normal tissues (4750), support the concept that lung eosinophils in acute TPE release mediators that are responsible, at least in part, for many of the symptoms and pathophysiologic abnormalities observed in this disorder.

Consistent with the hypothesis that the intense eosinophilic alveolitis of acute TPE is responsible, at least in part, for the derangements to the lower respiratory tract in this disease is the observation that when individuals with acute TPE are treated with DEC the improvement in lung function is concurrent with the resolution of the intense eosinophilic inflammation in the lower respiratory tract. However, it is interesting to speculate that, although the eosinophil is capable of injuring the normal lung parenchyma, there may be a threshold in the eosinophil burden to the lower respiratory tract below which the lung parenchyma can handle the "injurious" inflammatory potential of the eosinophil. In this context, the intense eosinophilic infiltration of acute TPE appears to be far less damaging to the alveolar structures than the more mild neutrophilic inflammation of a disorder such as idiopathic pulmonary fibrosis in which the derangements to the lower respiratory tract are severe (37).

\section{DEC therapy of acute TPE}

$\mathrm{DEC}$ is a piperazine derivative widely used in the treatment of filarial infections (58). Most individuals with acute TPE respond to the DEC therapy within 1 to 3 wk with a progressive reduction in symptoms, clearing of the chest $x$-ray, reduction in the blood eosinophil count, and improvement in lung function $(5,7,8$, $58,59)$. After therapy with DEC, there is a reduction in serum IgE and filarial specific IgG which occurs more gradually $(58,59)$.

The mechanisms of action of DEC are unclear. In vitro it is inactive against microfilariae (60), although it is generally appreciated to be "opsonic" for microfilariae in vivo causing their rapid clearance from the blood of microfilaremic individuals (58) and to be filaricidal against both microfilariae and the adult worms in vivo $(58,60)$. Other in vitro studies also suggesting that DEC acts to augment inflammatory processes directed against the microfilariae include the demonstration that DEC enhances the adherence of eosinophils to microfilariae $(61,63)$, activates complement associated with microfilariae (64), generally stimulates granulocyte adherence $(62,63)$, and likely stimulates eosinophils to release preformed granule proteins such as major basic protein onto the surface of microfilariae (65).

Despite the fact that how DEC acts in acute TPE is not fully understood, the present study suggests at least one consequence of DEC therapy in this disease is the suppression of the intense eosinophilic alveolitis. Since eosinophils can clearly injure the normal lung parenchyma, this observation is consistent with the concept that the improvement in symptoms, $x$-ray and lung function observed in individuals with acute TPE treated with DEC results, at least in part, from the direct and/or indirect suppression of the exaggerated eosinophilic inflammation of the lower respiratory tract that characterizes this disorder.

\section{Acknowledgments}

We would like to acknowledge the valuable support given by the staffs of the Tuberculosis Research Center and the Madras General Hospital (Madras), the Indian Council of Medical Research and Department of Science and Technology of the government of India (New Delhi). This study was carried out as part of the Indo-U. S. Science and Technology Initiative.

\section{References}

1. Neva, F. A., and E. A. Ottesen. 1978. Tropical (filarial) eosinophilia. N. Engl. J. Med. 298:1129-1131.

2. Ottesen, E. A. 1984. Immunological aspects of lymphatic filariasis and onchocerciasis. Trans. R. Soc. Trop. Med. Hyg. 73(Suppl.):9-18.

3. Piessens, W. F., and C. D. MacKenzie. 1982. Immunology of lymphatic filariasis and onchocerciasis. In Immunology of Parasitic Infections. S. G. Cohen and K. S. Warren, editors. Blackwell Scientific Publications, St. Louis. 622-653.

4. Ottesen, E. A. 1980. Immunopathology of lymphatic filariasis in man. Springer Semin. Immunopathol. 2:373-385.

5. Spry, C. J. F., and V. Kumaraswami. 1982. Tropical eosinophilia. Semin. Hematol. 19:101-115.

6. Udwadia, F. E. 1967. Tropical eosinophilia: a correlation of clinical, histopathologic and lung function studies. Dis. Chest. 52:531-538.

7. Poh, S. C. 1974. The course of lung function in treated tropical pulmonary eosinophilia. Thorax. 29:710-712.

8. Kamat, S. R., N. V. U. Warrier, S. D. Store, E. D. Kavandikar, and V. R. Hoskote. 1976. Clinical studies in pulmonary eosinophilia. I. Comparative study of response to diethylcarbamazine and corticosteroid drugs. Ind. J. Chest Dis. 18:221-232.

9. Udwadia, F. E. 1975. Tropical eosinophilia. In Pulmonary Eosinophilia: Progress in Respiration Research. S. A. Karger, Basel. 7:35-155.

10. Webb, J. K. G., C. K. Job, and E. W. Gault. 1960. Tropical eosinophilia: demonstration of microfiliariae in lung, liver and lymphnodes. Lancet. i:835-842.

11. Ottesen, E. A., F. A. Neva, R. S. Paranjape, S. P. Tripathy, K. V. Thiruvengadam, and M. A. Beaven. 1979. Specific allergic sensitisation to filarial antigens in tropical eosinophilia syndrome. Lancet. i: $1158-1161$.

12. Danaraj, T. J., G. Pacheco, K. Shanmugaratnam, and P. C. Beaver. 1966. The etiology and pathology of eosinophilic lung (tropical eosinophilia). Am. J. Trop. Med. Hyg. 15:183-189.

13. Joshi, U. V., F. E. Udwadia, and R. K. Gadgil. 1969. Etiology of tropical eosinophilia: a study of lung biopsies and review of published reports. Am. J. Trop. Med. Hyg. 18:231-240.

14. Hussain, R., R. G. Hamilton, V. Kumaraswami, F. Adkinson, and E. A. Ottesen. 1986. IgE responses in human filariasis. I. Quantitation of filaria-specific IgE. J. Immunol. 127:1623-1629. 
15. Nutman, T. B., E. A. Ottesen, A. S. Fauci, and D. J. Volkman. 1984. Parasite antigen-specific human $T$ cell lines and clones: major histocompatibility complex restriction and B cell helper function. J. Clin. Invest. 73:1754-1762.

16. Fulmer, J. D., W. C. Robert, E. R. von Gal, and R. G. Crystal. 1977. Small airways in idiopathic pulmonary fibrosis: comparison of morphologic and physiologic observations. J. Clin. Invest. 60:595-610.

17. Cotes, J. E., J. M. Dabbs, A. M. Hall, S. C. Lakhera, M. J. Saunders, and M. S. Malhotra. 1975. Lung function of healthy young men in India: contributory roles of genetic and environmental factors. Proc. R. Soc. Lond. B. 191:413-425.

18. Kamat, S. R., N. K. Tyagi, and S. S. A. Rashid. 1982. Lung function in Indian adult subjects. Lung India. 1:11-21.

19. Hunninghake, G. W., J. E. Gadek, O. Kawanami, V. J. Ferrans, and R. G. Crystal. 1979. Inflammatory and immune processes in the human lung in health and disease: evaluation by bronchoalveolar lavage. Am. J. Pathol. 97:149-206.

20. Saltini, C., A. J. Hance, V. J. Ferrans, F. Basset, P. B. Bitterman, and R. G. Crystal. 1984. Accurate quantification of cells recovered by bronchoalveolar lavage. Am. Rev. Respir. Dis. 130:650-658.

21. Rennard, S. I., G. Bassett, D. Lecossier, K. M. O'Donnell, P. Pinkston, P. G. Martin, and R. G. Crystal. 1986. Estimation of volume of epithelial lining fluid recovered by lavage using urea as a marker of dilution. J. Appl. Physiol. 60:532-538.

22. Weingarten, R. J. 1943. Tropical eosinophilia. Lancet. i:103105.

23. Spencer, H. 1977. The anatomy of the lung. In H. Spencer, editor. Pathology of the Lung: excluding pulmonary tuberculosis. Pergamon Press Inc., Elmsford, NY. 15-69.

24. Hunninghake, G. W., O. Kawanami, V. J. Ferrans, R. C. Young, W. C. Roberts, and R. G. Crystal. 1981. Characterization of the inflammatory and immune effector cells in the lung parenchyma of patients with interstitial lung disease. Am. Rev. Respir. Dis. 123:407-412.

25. Haslam, P. L.; C. W. G. Turton, B. Heard, A. Lukoszek, J. V. Collins, A. J. O. Salsbury, and M. Turner-Warwick. 1980. Bronchoalveolar lavage in pulmonary fibrosis: comparison of cells obtained with lung biopsy and clinical features. Thorax. 35:9-18.

26. Cohen, S. G., and E. A. Ottesen. 1983. The eosinophil, eosinophilia and eosinophil-related disorders. In Allergy: Principles and Practice. E. Middleton, C. E. Reed, and E. F. Ellis, editors. Mosby, St. Louis. 2: 701-769.

27. Goetzl, E. J., and K. F. Austen. 1975. Purification and synthesis of eosinophilotactic tetrapeptides of human lung tissue: identification as eosinophil chemotactic factor of anaphylaxis. Proc. Natl. Acad. Sci. USA. 72:4123-4127.

28. Clark, R. A. F., J. I. Gallin, and A. P. Kaplan. 1975. The selective eosinophil chemotactic activity of histamine. J. Exp. Med. 142:14621476.

29. Cohen, S., and P. A. Ward. 1971. In vitro and in vivo activity of a lymphocyte and immune-complex dependent chemotactic factor for eosinophils. J. Exp. Med. 133:133-146.

30. Colley, D. G. 1973. Eosinophils and immune mechanisms. I Eosinophil stimulation promoter (ESP): a lymphokine induced by specific antigen or phytohemagglutinin. J. Immunol. 110:1419-1423.

31. Tsuda, S., K. Fukuyama, and W. L. Epstein. 1980. Eosinophil response in guinea pig skin to a low molecular weight, eosinophil chemotactic factor extracted from livers of mice with schistosomiasis. $J$. Invest. Dermatol. 74:216-218.

32. Wadee, A. A., and R. Sher. 1980. The effects of soluble factor released by sensitized mononuclear cells incubated with $\mathrm{S}$. haematobium ova on eosinophil migration. Immunology. 41:989-995.

33. Czarnetski, B. M. 1981. In vitro generation of eosinophil chemotactic factor (ECF) from human and murine mononuclear phagocytes. Scand. J. Immunol. 13:511-516.

34. Basset, P., A. B. Tonel, M. Joseph, L. Prin, A. Mallart, J. Charon, and A. Capron. 1984. Secretion of a chemotactic factor for neutrophils and eosinophils by alveolar macrophages from asthmatic patients. $J$. Allergy Clin. Immunol. 74:827-834.
35. Kay, A. B. 1970. Studies on eosinophil leukocyte migration. I. Factors specifically chemotactic for eosinophils and neutrophils generated from guinea pig serum by antigen-antibody complexes. Clin. Exp. Immunol. 7:723-737.

36. Tanaka, J., T. Baba, and M. Torisu. 1979. Ascaris and eosinophils. I. Isolation and characterization of eosinophil chemotactic factor and neutrophil chemotactic factor of parasite in Ascaris antigen. J. Immunol. 122:302-308

37. Crystal, R. G., P. B. Bitterman, S. I. Rennard, A. J. Hance, and B. A. Keogh. 1984. Interstitial lung disease of unknown etiology: disorders characterized by chronic inflammation of the lower respiratory tract. $N$. Engl. J. Med. 310:154-166, 235-244.

38. Davis, W. B., G. A. Fells, X. Sun, J. E. Gadek, A. Venet, and R. G. Crystal. 1984. Eosinophil-mediated injury to lung parenchymal cells and interstitial matrix. J. Clin. Invest. 74:269-278.

39. Godard, P., J. Chaintreuil, M. Damon, M. Coupe, O. Flandre, A. Crastes dePaulet, and F. B. Michel. 1982. Functional assessment of alveolar macrophages: comparison of cells from asthmatics and normal subjects. J. Allergy Clin. Immunol. 70:88-93.

40. Weller, P. F., and E. J. Goetzl. 1980. The human eosinophil. Roles in host defense and tissue injury. Am. J. Pathol. 100:793-820.

41. Gleich, G. J., and D. A. Loegering. 1984. Immunobiology of eosinophils. Annu. Rev. Immunol. 2:429-459.

42. Ayers, G. H., L. C. Altman, G. H. Glerich, D. A. Loegering, and C. B. Baker. 1985. Eosinophil and eosinophil granule-mediated pneumocyte injury. J. Allergy Clin. Immunol. 76:595-604.

43. Klebanoff, S. J., W. R. Henderson, E. C. Jong, A. Jörg, and R. M. Locksley. 1983. Role of Peroxidase in Eosinophil Function. In Immunobiology of the Eosinophil. T. Yoshida, and M. Torisu, editors. Elsevier/North Holland, Inc., Amsterdam. 261-282.

44. Connell, J. T. 1968. Morphological changes in eosinophils in allergic disease. J. Allergy. 41:1-9.

45. Tai, P. C., and C. J. F. Spry. 1976. Studies on blood eosinophils. I. Patients with a transient eosinophilia. Clin. Exp. Immunol. 24:415422.

46. Kay, A. B. 1985. Eosinophils as effector cells in immunity and hypersensitivity disorders. Clin. Exp. Immunol. 62:1-12.

47. Frigas, E., D. A. Loegering, and G. J. Gleich. 1980. Toxic effects of the guinea pig eosinophil major basic protein on tracheal epithelium. Lab. Invest. 42:35-43.

48. Gleich, G. J., E. Frigas, D. A. Loegering, D. L. Wassom, and D. Steinmuller. 1979. Cytotoxic properties of the eosinophial major basic protein. J. Immunol. 123:2925-2927.

49. Frigas, E., D. A. Loegering, G. O. Solley, G. M. Farrow, and G. J. Gleich. 1981. Elevated levels of the eosinophil granule major basic protein in the sputum of patients with bronchial asthma. Mayo Clin. Proc. 56:345-353.

50. Filley, W. V., G. M. Kephart, K. E. Holley, and G. J. Gleich. 1982. Identification by immunofluorescence of eosinophil granule major basic protein in lung tissues of patients with bronchial asthma. Lancet. ii: $11-15$.

51. Worden, K. A., W. J. Metzger, W. Kopp, H. B. Richerson, and G. W. Hunninghake. 1983. Dissolution of eosinophil granules in bronchial lavage obtained from allergic asthmatics during bronchoprovocation and seasonal exposure. Proc. Ann. EMSA. 41:789-790.

52. McEvoy, J. D. S., K. J. Donald, and R. L. Edwards. 1978. Immunoglobulin levels and electron microscopy in eosinophilic pneumonia. Am. J. Med. 64:529-536.

53. Fox, B., and W. A. Seed. 1980 . Chronic eosinophilic pneumonia. Thorax. 35:570-580.

54. Grantham, J. G., J. A. Meadows, and G. J. Gleich. 1986. Chronic eosinophilic pneumonia. Evidence for eosinophil degranulation and release of major basic protein. Am. J. Med. 80:89-94.

55. Kanner, R. E., and S. P. Hammar. 1977. Chronic eosinophilic pneumonia. Ultrastructural evidence of marked immunoglobulin production plus macrophagic ingestion of eosinophils and eosinophilic lysosomes leading to intracytoplasmic Charcot-Leyden crystals. Chest. 71: 95-98. 
56. Fauci, A. S., J. B. Harley, W. C. Roberts, V. J. Ferrans, H. R. Gralnick, and B. H. Bjornson. 1982. The idiopathic hypereosinophilic syndrome: clinical, pathophysiologic and therapeutic considerations. Ann. Intern. Med. 97:78-92.

57. Songsiridej, V., P. J. Dor, Petersons, S. J. Ackerman, G. J. Gleich, and W. W. Busse. 1985. Facial edema and eosinophilia. Evidence for eosinophil degranulation. Ann. Intern. Med. 103:503-506.

58. Ottesen, E. A. 1985. Efficacy of diethylcarbamazine in eradicating infection with the lymphatic dwelling filariae of humans. Rev. Infect. Dis. 7:341-356.

59. Neva, F. A., A. P. Kaplan, G. Pacheco, L. Gray, and J. Danaraj. 1975. Tropical eosinophilia: a human model of parasitic immunopathology, with observations on serum IgE levels before and after treatment. J. Allergy Clin. Immunol. 55:422-429.

60. Piessens, W. F., and M. Beldaekas. 1979. Diethylcarbamazine enhances antibody-mediated cellular adherence to Brugia malayi microfilariae. Nature (Lond.). 282:845-847.
61. Racz, P., K. Tenner-Racz, D. W. Buttner, and E. J. Albiez. 1982. Ultrastructural evidence for eosinophil parasite adherence (EPA) reaction in human onchocercal lymphadenitis in the early period following diethylcarbamazine treatment. Tropenmed. Parasit. 33:213-218.

62. King, C. H., B. M. Greene, and P. J. Spagnuolo. 1983. Diethylcarbamazine citrate, an antifilarial drug, stimulates granulocyte adherence. Antimicrob. Agents Chemo. 24:453-456.

63. Greene, B. M., H. R. Taylor, and M. Aikawa. 1981. Cellular killing of microfiliariae of Onchocerca volvulus: eosinophil and neutrophilmediated immune serum dependent destruction. J. Immunol. 127:16111618.

64. Staniunas, R. J., and B. Hammerberg. 1982. Diethylcarbamazineenhanced activation of complement by intact microfilariae of Dirofilaria immitis and their in vitro products. J. Parasitol. 68:809-816.

65. Kephart, G. M., G. J. Gleich, D. H. Connor, D. W. Gibson, and S. J. Ackerman. 1984. Deposition of eosinophil granule major basic protein on the microfilariae of Onchocerca volvulus in the skin of patients treated with diethylcarbamazine. Lab. Invest. 50:51-61. 